\title{
Correction to: Coercive Parenting Mediates the Relationship between Military Fathers' Emotion Regulation and children's Adjustment
}

\author{
Jingchen Zhang ${ }^{1}$ - Alyssa Palmer ${ }^{2} \cdot \mathrm{Na}_{\text {Zhang }}{ }^{3} \cdot$ Abigail H. Gewirtz $^{4}$
}

Published online: 25 April 2020

(C) Springer Science+Business Media, LLC, part of Springer Nature 2020

\section{Correction to: Journal of Abnormal Child Psychology} https://doi.org/10.1007/s10802-020-00625-8

The original version of this article unfortunately contained a mistake. The formatting of Table 2 is distorted. The corrected table is given below.

The online version of the original article can be found at https://doi.org/ $10.1007 / \mathrm{s} 10802-020-00625-8$

$\triangle \quad$ Abigail H. Gewirtz agewirtz@umn.edu

1 Department of Family Social Science, University of MinnesotaTwin Cities, Minneapolis, MN, USA

2 Institute of Child Development, University of Minnesota-Twin Cities, Minneapolis, MN, USA

3 REACH Institute, Department of Psychology, Arizona State University, Minneapolis, MN, USA

4 Department of Family Social Science and Institute of Child Development \& Institute of Translational Research in Children's Mental Health, University of Minnesota-Twin Cities, 290 McNeal Hall, 1985 Buford Avenue, St. Paul, MN 55108, USA 
Table 2 Model fit indices for the direct effects, indirect effects, and the 95\% confidence interval for the indirect effects

\begin{tabular}{|c|c|c|c|c|c|c|c|c|c|}
\hline \multirow[t]{2}{*}{ Models } & \multirow[t]{2}{*}{ Outcome variable } & \multirow[t]{2}{*}{$\chi 2(d f)$} & \multirow[t]{2}{*}{ CFI } & \multirow[t]{2}{*}{ RMSEA } & \multirow[t]{2}{*}{ SRMR } & \multicolumn{4}{|c|}{ Indirect effects } \\
\hline & & & & & & $B$ & $S E$ & $\beta$ & $95 \% \mathrm{CI}$ \\
\hline \multirow[t]{3}{*}{ Direct effects } & Internalizing problems & $7.52(10)$ & 1.00 & .00 & .02 & & & & \\
\hline & Externalizing problems & $7.92(10)$ & 1.00 & .00 & .02 & & & & \\
\hline & Self-reported depression & $10.68(10)$ & 1.00 & .02 & .03 & & & & \\
\hline \multirow{3}{*}{$\begin{array}{l}\text { Indirect effects through } \\
\text { coercive parenting }\end{array}$} & Internalizing problems & $49.77(34)$ & .96 & .05 & .03 & -.11 & .06 & -.07 & {$[-.28,-.02]$} \\
\hline & Externalizing problems & $55.70(34)$ & .95 & .06 & .03 & -.14 & .07 & -.07 & {$[-.31,-.03]$} \\
\hline & Self-reported depression & $43.57(33)$ & .96 & .05 & .04 & -.02 & .01 & -.11 & {$[-.42,-.00]$} \\
\hline
\end{tabular}

$95 \% \mathrm{CI}=95 \%$ confidence internals derived from the bias-corrected bootstrapping with 2000 iterations

Publisher's Note Springer Nature remains neutral with regard to jurisdictional claims in published maps and institutional affiliations. 